\title{
Using silicon-vacancy centers in diamond to probe the full strain tensor
}

\author{
Kelsey M. Bates, ${ }^{1}$ Matthew W. Day, ${ }^{1}$ Christopher L. Smallwood, ${ }^{1,2}$ Rachel C. Owen, ${ }^{1}$ Ronald Ulbricht, ${ }^{3}$ Tim \\ Schröder, ${ }^{4,5}$ Edward Bielejec, ${ }^{6}$ and Steven T. Cundiff, ${ }^{1, a)}$ \\ ${ }^{1)}$ Department of Physics, University of Michigan, Ann Arbor, MI 48109, USA \\ ${ }^{2)}$ Department of Physics and Astronomy, San José State University, San José, CA 95192, \\ USA \\ ${ }^{3)}$ Department of Molecular Spectroscopy, Max Planck Institute for Polymer Research, Mainz, 55128, \\ Germany \\ 4) Department of Electrical Engineering and Computer Science, Massachusetts Institute of Technology, Cambridge, MA 02138, \\ USA \\ ${ }^{5)}$ Department of Physics, Humboldt-Universität zu Berlin, Newtonstrasse 15, 12489 Berlin, \\ Germany \\ ${ }^{6)}$ Sandia National Laboratories, Albuquerque, NM 87185, USA
}

(Dated: 21 April 2021)

An ensemble of silicon vacancy centers in diamond $\left(\mathrm{SiV}^{-}\right)$is probed using two coherent spectroscopy techniques. Two main distinct families of $\mathrm{SiV}^{-}$centers are identified using multidimensional coherent spectroscopy, and these families are paired with two orientation groups by comparing spectra from different linear polarizations of the incident laser. By tracking the peak centers in the measured spectra, the full diamond strain tensor is calculated local to the laser spot. Such measurements are made at multiple points on the sample surface and variations in the strain tensor are observed.

\section{INTRODUCTION}

Color centers in diamond and other crystalline host materials have shown potential in recent years in applications as diverse as remote magnetic field sensing and imaging $1+4$, as room-temperature qubit 5 , as single photon sources 6 , and even as candidates for use in novel particle detection schemes in dark matter direct detection experiment $\$ 10 / 11$. One such color center is the negatively charged silicon-vacancy center $\left(\mathrm{SiV}^{-}\right)$in diamond. Composed of an interstitial silicon atom located between two carbon vacancies in the diamond lattice, $\mathrm{SiV}^{-}$centers are, to a large extent, protected from first-order perturbations by the similarity of their ground and excited states ${ }^{12}$. As a result, their electrostatic dipole moment vanishes, which reduces the $\mathrm{SiV}^{-}$sensitivity to first-order perturbations. In addition, $\mathrm{SiV}^{-}$centers exhibit weak electronphonon coupling $\frac{13}{13}-16$ relative to other types of color centers in diamond. These properties combine to increase dephasing times of the $1.68 \mathrm{eV}$ zero phonon line (ZPL) transition manifold to greater than $20 \mathrm{~ns}$ in some cases ${ }^{17}$. Due to the robustness and isolation which the diamond lattice provides, the $\mathrm{SiV}^{-}$is a promising candidate for use in many quantum information technology, remote sensing, and photonics applications.

In addition to these applications, it has recently been demonstrated that color centers in diamond can be used to sense strain in their environments $\frac{18}{20}$ by carefully tracking the frequencies of various optical or magnetic resonances as a function of sample position, and then mapping that data to strain. This sensitivity is potentially useful in applications where remotely measuring the deviation of structural components from expected stresses and strains is desired. For example, due to the ubiquity of diamond anvil cells as devices

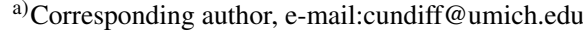

to apply strain to study a wide variety of material samples ${ }^{21}$, having direct color center strain imaging probes in situ would represent a tremendous advantage over traditional strain estimation techniques when studying small volume samples.

To this end, we demonstrate a method employing siliconvacancy centers in diamond to spectroscopically map all strain tensor elements. We employ two different coherent spectroscopic techniques to track the influence of strain on the $\mathrm{SiV}^{-}$ zero phonon line transitions in our sample. By combining linear spectra with nonlinear, multi-dimensional spectra2223 as a function of polarization, we are able to provide an estimate of the ensemble averaged strain in the presence of inhomogenaiety in our sample. Our methods are widely applicable and easily replicable with the recent advent of commercially available coherent spectrometers.

\section{EXPERIMENTAL METHODS}

\section{A. Spectroscopic Techniques}

We study an ensemble of $\mathrm{SiV}^{-}$centers with two different coherent spectroscopy techniques. The first is a two-pulse correlation (TPC) measurement. Here, two collinear optical pulses from a Ti:Sapph laser, which are frequency tagged with acousto-optic modulators in a Mach-Zhender interferometer, are sent to the sample, as depicted in Fig. 11(a). Using a lockin amplifier, we measure the modulated photoluminescence from the sample as a function of the temporal separation $(t)$ between the pulses. Additionally, a continuous wave laser is co-propagated with the Ti:Sapph pulses to act as a phase reference for the lock-in amplifier and to remove the effects of mechanical fluctuations in the experiment. The photoluminescence intensity is Fourier transformed with respect to $t$, to yield a one-dimensional, coherently-detected absorption spectrum. The phase coherent spectra reject signal contributions from long-timescale effects, as the spectral response is 
recorded as a function of time delays between pulses which vary between $100 \mathrm{fs}$ and $1 \mathrm{~ns}$.

a)

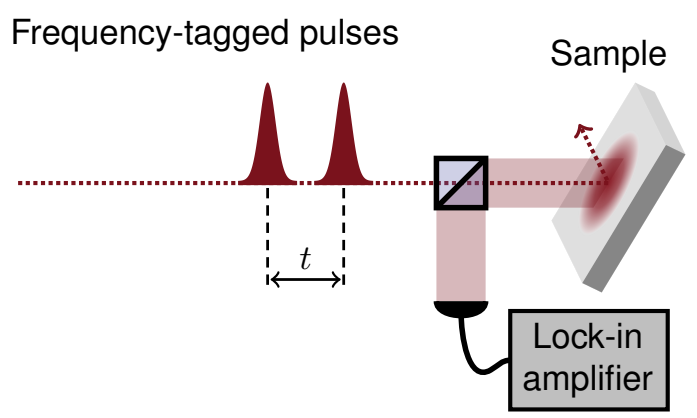

b)

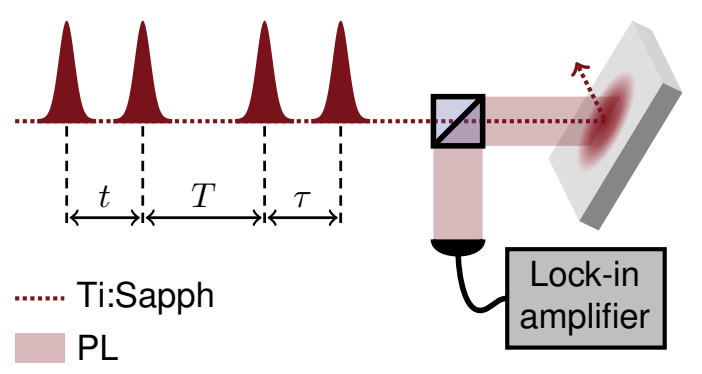

FIG. 1. A depiction of the experimental setup, including both (a) two-pulse correlation (TPC) measurements and (b) multidimensional coherent spectroscopy (MDCS) measurements.

For this sample, the linear TPC spectra can be difficult to interpret on their own, as there are many observed peaks to identify. Thus, we also employ a more powerful third-order nonlinear spectroscopic technique, multidimensional coherent spectroscopy (MDCS). Our collinear MDCS variant2425], depicted in Fig. 1. (b), uses a nested Mach-Zhender interferometer to separate four optical pulses from the Ti:Sapph laser. For the spectra used here, the first $(\tau)$ and third $(t)$ time delays are varied, and these data are Fourier transformed with respect to these time delays to obtain a two dimensional map of the thirdorder signal as a function of excitation $\left(\omega_{\tau}\right)$ and detection $\left(\omega_{t}\right)$ frequency axe ${ }^{2425}$. Note that because $\omega_{t}$ has opposite phase evolution from $\omega_{\tau}$, the vertical excitation axis is negative, thus the diagonal at which the excitation and detection frequencies have equal magnitude is the downward sloping diagonal.

There are two main advantages to using multidimensional spectra. First, we can easily distinguish between homogeneous and inhomogeneous broadening, as these will broaden the MDCS peak along perpendicular diagonal directions. By measuring the widths of the peaks along and across the equal frequency diagonal, we can measure the both the homogeneous and inhomogeneous linewidths respectively. More important to this work, MDCS spectra can be used to identify if two spectral peaks are coupled. If coupling exists between two transitions, a coupling crosspeak will occur where the excitation and detection frequencies correspond to those of the two coupled transitions. Since a single $\mathrm{SiV}^{-}$center can emit at four different frequencies, a spectrum with several shifted families of $\mathrm{SiV}^{-}$centers can be difficult to interpret. Using a single multidimensional spectrum, we can determine which spectral peaks correspond to a single family.

While the information contained in a TPC measurement is not as rich as that in a full MDCS spectrum, the main advantage is that the acquisition time for a TPC spectrum is much shorter. This enables us to take a large number of linear spectra to observe position dependent trends, while only taking a few select MDCS spectra when needed to assign the peaks.

As mentioned previously, both the TPC and MDCS spectra used here utilize collinear geometries. This enables a smaller spot size than $k$-vector selection MDCS experiments ${ }^{25}$, and allows us to compare spectra taken at nearby locations on the sample. Studies of color centers often take measurements of a single center, with no guarantees that the center is representative of neighboring centers. In contrast, the high density of our sample and the ability of MDCS to untangle complicated spectra enable us to accurately measure the ensemble averaged optical response.

For both of these spectroscopic techniques, we linearly polarized the light incident on our sample. This was mainly so that the detected light could be cross-polarized to reduce laser scatter. Additionally, since the four electronic transitions of the $\mathrm{SiV}^{-}$center are polarization dependen ${ }^{8}$, spectra taken at each polarization can be compared to understand the geometry of the sample.

\section{B. Sample Information}

Our sample is a chemical-vapor deposition grown, [110]oriented mono crystalline diamond. An ensemble of $\mathrm{SiV}^{-}$ centers was created by implanting Silicon-29 ions with a focused ion beam, at a depth of $0.5-2.4 \mu \mathrm{m}$ and number density $7.5 \times 10^{18} \mathrm{~cm}^{-3}$. The sample was then annealed at $1000-1050^{\circ} \mathrm{C}$ and tri-acid cleaned. A picture of the sample is included in Fig. 3 (a). The sample has been cleaved, which explains its unusual shape.

$\mathrm{SiV}^{-}$centers can occur along four different orientations in diamond, corresponding to the four directions of carboncarbon bonds in the diamond lattice. Figure 2 (b) shows the four orientations of $\mathrm{SiV}^{-}$centers in our [110]-oriented sample, where the four vectors on the diamond correspond to the $\mathrm{SiV}^{-}$axis shown in Fig. 2 (a). Note that we can group these peaks into in-plane (orange) and out-of-plane (purple) orientation families.

Figure 2 (c) depicts the level structure of the $\mathrm{SiV}^{-}$zerophonon line (ZPL). The ZPL has mean frequency $\Delta_{\mathrm{ZPL}}=$ $407 \mathrm{THz}$, and the frequencies of the four optical transitions are also determined by the ground state and excited state splittings, $\Delta_{\mathrm{gs}}$ and $\Delta_{\mathrm{es}}$. It has been previously shown that two of the optical transitions are excited by light polarized perpendicular to the the $\mathrm{SiV}^{-}$axis shown in Fig. 2 (a), and and the other two are excited by light polarized parallel to this axis 8 .

The sample was placed in a closed-loop cryostat at a temperature of $11 \mathrm{~K}$, and the optical experiments were conducted using a home-built confocal microscope to focus $736 \mathrm{~nm}$ pulses from a Titanium:Sapphire (Ti:Sapph) oscillator onto the sample face. As shown in Fig. 1) the sample is tilted by $30^{\circ}$ from normal to reject the reflected Ti:Sapph beam and 
any coherent scatter that could corrupt the PL measurements. The two linear polarizations of light used here are depicted in Fig]2(b).

\section{RESULTS AND ANALYSIS}

\section{A. Peak Identification}

Previous experimental data taken of $\mathrm{SiV}^{-}$centers $8[20|27| 28$ shows the four spectral peaks corresponding to the four optical transitions in Fig. 2 (c). Incoherent photoluminescence spectra taken from our sample has this structure as well 28 . However, the TPC photoluminescence spectrum in Fig. 2 (d) shows significantly more peaks, indicating the presence of multiple groups of color centers.

The TPC measurement contains no information on coherent coupling between states. Additionally, due to the large strain susceptibility of the system, small variations in strain could change the ground state splittings on the order of $10 \mathrm{GHz}^{20}$, making it difficult to tell which transitions belong to the same groups of color centers.

MDCS can be used to identify the groups of peaks in the spectra. Spectra taken with horizontally and vertically incident light were summed to create the spectrum in Fig. 2 (e). The insets (f-h) below this spectrum show more detail for some of the crosspeaks, both for the two linear spectra and the combined spectrum. Note that in the combined spectrum, the seven visible crosspeaks can be grouped into two squares, which are highlighted in (f). Each square is formed from two lower energy crosspeaks, and two higher energy crosspeaks, and the peaks used in each square are distinct. This allows us to group the spectral peaks into two families, where peaks in a single family are coupled, and no coupling is observed between different families. Using this, we conclude that each family of peaks corresponds to a different type of $\mathrm{SiV}^{-}$center. Additionally, the MDCS spectrum shows some inhomogeneity, which could be due to microscopic strain fluctuations ${ }^{28}$ or interactions between nearby $\mathrm{SiV}^{-}$centers 29 .

To identify the origin of the two families of $\mathrm{SiV}^{-}$centers, we can use the polarization data. While the MDCS spectra can be used for this, it is easier to compare linear spectra, such as the TPC spectra shown in Fig. 2(d). Note that the relative peak strengths of the two families of peaks under different polarizations of incident light are very different. This suggests the two families of $\mathrm{SiV}^{-}$centers are oriented differently in the diamond lattice. By relating the different possible orientations of $\mathrm{SiV}^{-}$centers in our sample to the polarization selection rules shown in Fig. 22(c), we find that the two peak families identified above correspond to the in-plane and out-of-plane orientations in Fig 2(b). While it may be feasible to group the peaks into families based solely on polarization data, this would become increasingly difficult for higher amounts of strain, and the crosspeaks in the MDCS spectrum are easier to interpret and give a higher degree of certainty.

The peaks corresponding to these families are indicated in Fig. 2 (d-e) using orange and purple vertical lines, corresponding to the in-plane and out-of-plane families. In the scans seen here, we do see additional splitting of the in-plane peaks. The specific in-plane orientation can be determined with additional linearly polarized spectra.

\section{B. Strain Calculation}

While Section III A partially explains our spectra, it does not explain the origin of the peak shifts. We propose that the source of these shifts is due to strain intrinsic to our sample, mainly because the shifts are different for different $\mathrm{SiV}^{-}$orientations. By assuming this hypothesis is true, we can solve for the full strain tensor in our sample.

Previous work completed by Meesala et al. 20 derived equations relating four strain susceptibility parameters to the six strain tensor indices local to a given $\mathrm{SiV}^{-}$center. They combined these equations with experimental data and simulated strain tensor data to fit for the strain susceptibility parameters. In this work, we instead used experimental data and the strain susceptibility parameters to estimate the strain tensor.

The equations from Ref. 20 are

$$
\begin{array}{r}
\Delta_{\mathrm{ZPL}}=\Delta_{\mathrm{ZPL}, 0}+\left(t_{\|, \mathrm{es}}-t_{\|, \mathrm{gs}}\right) \varepsilon_{z z}^{\prime}+\left(t_{\perp, \mathrm{es}}-t_{\perp, \mathrm{gs}}\right)\left(\varepsilon_{x x}^{\prime}-\varepsilon_{y y}^{\prime}\right), \\
\Delta_{\mathrm{gs}}=\sqrt{\lambda_{\mathrm{SO}, \mathrm{gs}}+4\left(d_{\mathrm{gs}}\left(\varepsilon_{x x}^{\prime}-\varepsilon_{y y}^{\prime}\right)+f_{\mathrm{gs}} \varepsilon_{z x}^{\prime}\right)+4\left(2 d_{\mathrm{gs}} \varepsilon_{x y}^{\prime}+f_{\mathrm{gs}} \varepsilon_{y z}^{\prime}\right)^{2}}, \\
\Delta_{\mathrm{es}}=\sqrt{\lambda_{\mathrm{SO}, \mathrm{es}}+4\left(d_{\mathrm{es}}\left(\varepsilon_{x x}^{\prime}-\varepsilon_{y y}^{\prime}\right)+f_{\mathrm{es}} \varepsilon_{z x}^{\prime}\right)+4\left(2 d_{\mathrm{es}} \varepsilon_{x y}^{\prime}+f_{\mathrm{es}} \varepsilon_{y z}^{\prime}\right)^{2}} .
\end{array}
$$

Note that Eqs. $1 \mathrm{~b}$ and $1 \mathrm{c}$ are linearly dependent, as the ground state and excited state splitting respond similarly to strain. These equations are given in the $\mathrm{SiV}^{-}$reference frame, which is depicted in Fig. 3 (b). Since there are four different orientations of $\mathrm{SiV}^{-}$centers in four distinct strain environments, we must first rotate these equations into a common basis. We choose the crystal basis depicted in Fig 3 (a). For instance, for one of the in-plane peaks we have

$$
\begin{aligned}
& \varepsilon_{x x}^{\prime}=\frac{1}{3} \varepsilon_{x x}-\frac{2 \sqrt{2}}{3} \varepsilon_{z x}+\frac{2}{3} \varepsilon_{z z}, \\
& \varepsilon_{y y}^{\prime}=\quad \varepsilon_{y y}, \\
& \varepsilon_{z z}^{\prime}=\frac{2}{3} \varepsilon_{x x}+\frac{2 \sqrt{2}}{3} \varepsilon_{z x}+\frac{1}{3} \varepsilon_{z z}, \\
& \varepsilon_{x y}^{\prime}=\frac{\sqrt{3}}{3} \varepsilon_{x y}-\frac{\sqrt{6}}{3} \varepsilon_{y z}, \\
& \varepsilon_{y z}^{\prime}=\frac{\sqrt{6}}{3} \varepsilon_{x y}+\frac{\sqrt{3}}{3} \varepsilon_{y z}, \\
& \varepsilon_{z x}^{\prime}=\frac{\sqrt{2}}{3} \varepsilon_{x x}-\frac{\sqrt{2}}{3} \varepsilon_{z z}-\frac{1}{3} \varepsilon_{z x} .
\end{aligned}
$$


a)

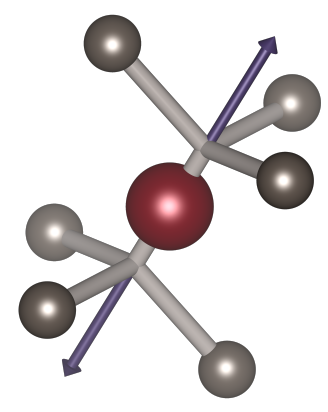

b)

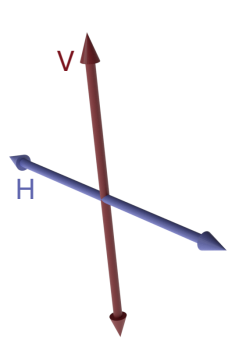

c)

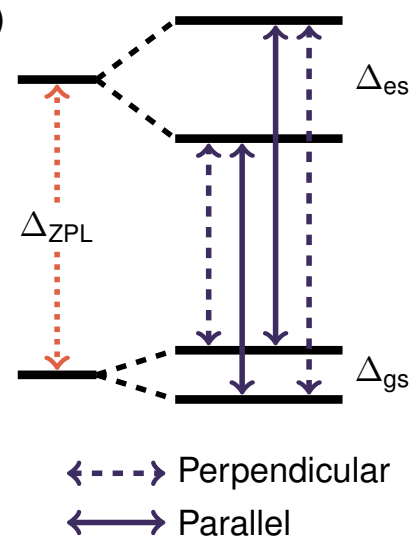

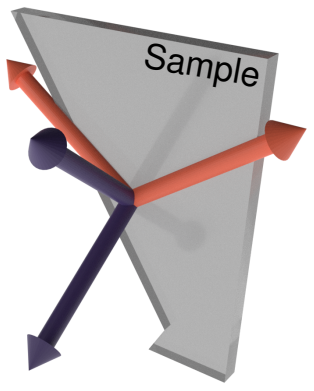

e)

d)
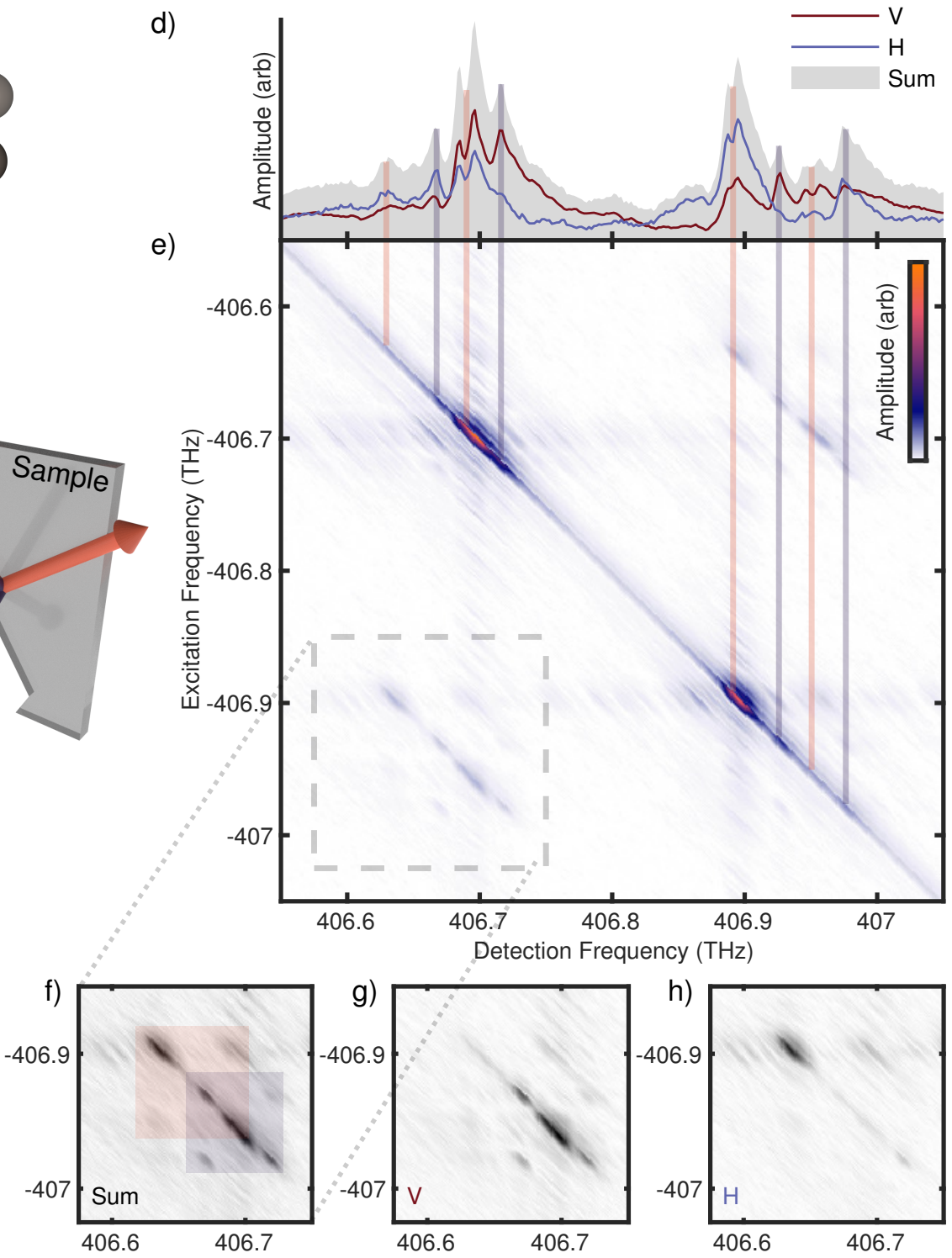

FIG. 2. (a) A depiction of the $\mathrm{SiV}^{-}$color cente ${ }^{26}$, with the red sphere representing a ${ }^{29} \mathrm{Si}$ interstitial atom between the two lattice vacancies, and the grey spheres representing nearby carbon atoms. The $\mathrm{SiV}^{-}$axis is shown in purple. (b) The four orientations of $\mathrm{SiV}^{-}$in our [110] oriented diamond. The orientations of the centers along the $\langle 111\rangle$ axes of the lattice yield two in-plane (orange) and two out-of-plane (purple) orientations. The directions of vertical and horizontal polarizations relative to the sample are shown. (c) $\mathrm{The}_{\mathrm{SiV}}^{-} \mathrm{ZPL}$ level structure, as well as the microscopic polarization selection rules with respect to the $\mathrm{SiV}^{-}$axis. (d) $\mathrm{TPC}$ spectra of the $\mathrm{SiV}^{-} \mathrm{ZPL}$ of vertical and horizontal polarizations, and their sum of the incident laser from a single point on the sample. (e) A rephasing, MDCS spectrum of the $\mathrm{SiV}^{-} \mathrm{ZPL}$, taken as the sum of spectra using both vertical and horizontal polarizations. The orange and purple vertical lines highlight the peaks corresponding to the two orientation families. (f-g) More detail of the outlined section in the MDCS spectrum, including both vertical and horizontal components. In (f), the crosspeak families are highlighted.

This calculation is repeated for the three other orientations, and the results are substituted into Eqs. 11, yielding twelve total equations, eight of which are independent. Since (in the crystal basis) there are six strain tensor indices, we can use these equations to solve for the full strain tensor.

We took a series of TPC spectra at 23 locations on our sample, as shown in Fig. 3 (c). These locations lie along a single line, with a gap in the middle where the implantation density is lower and reliable peak locations and strain estimates could not be measured. Slight shifts in the frequencies of the spectral peaks can be seen.

To use this data to solve for the strain, we first uses the techniques in Section III A to identify the peaks in our linear spectra (several MDCS spectra were taken at select locations to help with this). The peaks were fitted to find the locations of all 16 spectral peaks for each scan, taking advantage of both vertical and horizontal polarization spectra, since some peaks are more visible on a given polarization. In cases where two 
a)

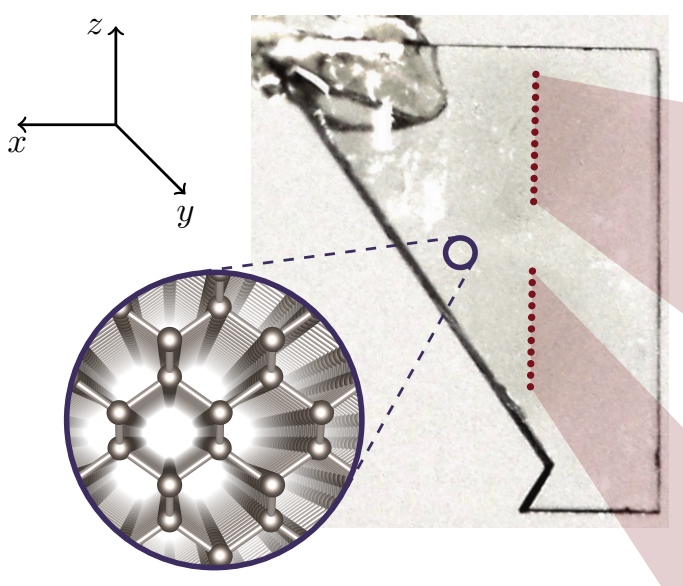

b)

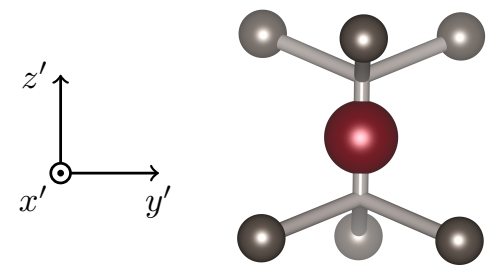

c)

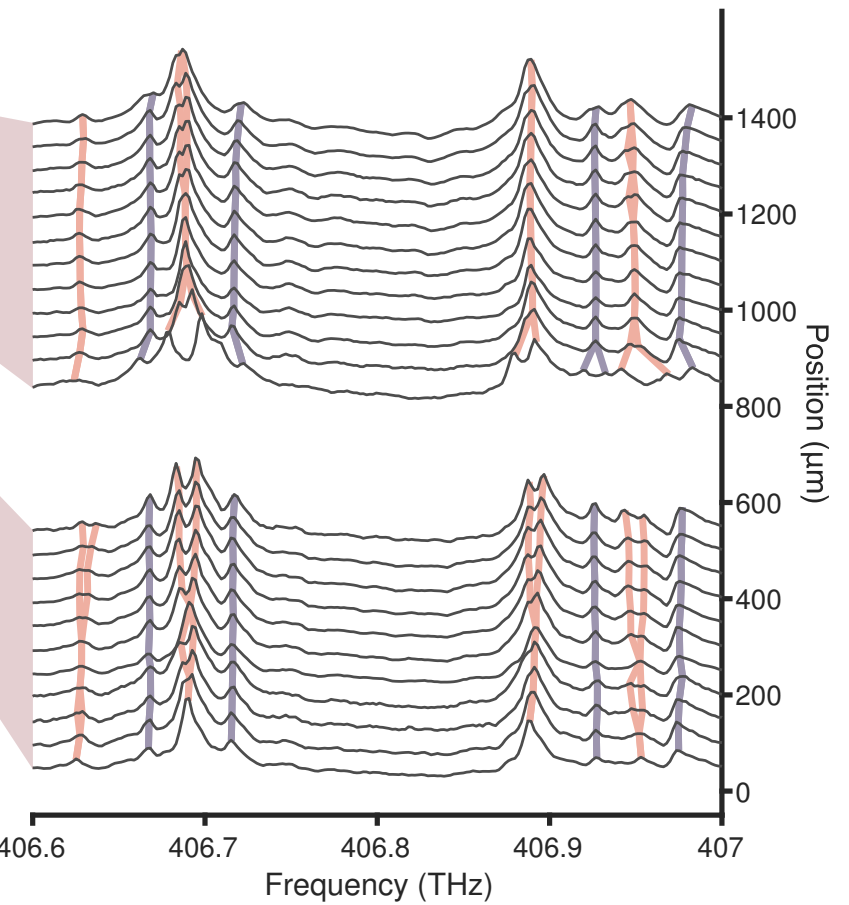

FIG. 3. (a) An image of the sample illustrating both the locations of the 23 linear spectra used in the strain calculation, as well as the crystal basis used for the global strain tensor. (b) The basis of a single $\mathrm{SiV}^{-}$center ${ }^{26}$. (c) A series of TPC spectra, summing both horizontal and vertical polarizations. The centers of each peak are highlighted.

peaks could not be resolved (which sometimes occurs for two in-plane or two out-of-plane peaks), a single frequency was reported.

Next, we used Eqs. 1 to find analytical expressions for the frequencies of the 16 spectral peaks as a function of the six strain tensor indices in the crystal basis, $p_{i}^{\text {calc }}(\varepsilon)$. We estimated the error in our measured peaks $p_{i}^{\text {meas }}$ to be $\sigma=2 \mathrm{GHz}$. This gives us an expression for $\chi^{2}$,

$$
\chi^{2}=\sum_{i=1}^{16} \frac{\left(p_{i}^{\text {meas }}-p_{i}^{\text {calc }}(\varepsilon)\right)^{2}}{\sigma^{2}} .
$$

Next we find the strain tensor $\varepsilon$, or equivalently the six strain tensor indices, such that $\chi^{2}$ is minimized. This tensor is our solved result.

To estimate the error in the calculation we note that if the error in our peak locations is random, then the $\chi^{2}$ value from Eqn. 3 should indeed follow a chi-square distribution with 16 degrees of freedom for the true value of the strain tensor. Thus, there is a $90 \%$ chance that $\chi^{2}<23.5$. We explored the parameter space local to our solved strain tensor to find the range of strain tensors such that $\chi^{2}$ is less than the desired value.

The strain solving algorithm was also tested on simulated data. Peaks were generated with Eqs. 1 using random values for the strain tensor indices. Random error was added to these peak values, and we attempted to recover the original strain tensor indices using the algorithm above. The algorithm worked as well as expected within the range of observed strain values. The algorithm begins to break down as the sheer strain tensor index $\varepsilon_{x y}$ becomes large (greater than $0.8 \times 10^{-5}$ ).

\section{Strain Results}

The results are shown in Fig. 4. Before drawing conclusions from these results, some limitations with the data should be addressed. First, the values of the normal strain tensor indices are dependent on the mean ZPL frequency at zero strain, or $\Delta_{\mathrm{ZPL}, 0}$. We did not take a direct measurement of this, but we estimated it to be $406.795 \mathrm{THz}$. However, we find that changing this value results in a constant offset of the normal strain tensor indices $\varepsilon_{x x}, \varepsilon_{y y}$ and $\varepsilon_{z z}$, and their relative values are preserved. Next, a sign ambiguity for small values of $\varepsilon_{x y}$ means we cannot find the sign of this parameter, so the plot in Fig. 4 uses positive $\varepsilon_{x y}$. For all indices, the error bars in Fig. 4 are only meant to represent error due to random error in the peak locations. They do not account for systematic errors, such as errors in the strain susceptibility parameters used in Eqs.1.

Despite these limitations, we do see a varying strain in our sample. The normal strain indices are nonzero, and these values appear to vary across the sample. While we do not see statistically significant magnitudes for the sheer strain tensor indices $\varepsilon_{x y}$ and $\varepsilon_{y z}$, the sheer strain tensor index $\varepsilon_{z x}$ does show statistically significant variation.

Previous strain measurements ${ }^{20}$ observe shifts in single $\mathrm{SiV}^{-}$centers. By contrast, these measurements are taken on 


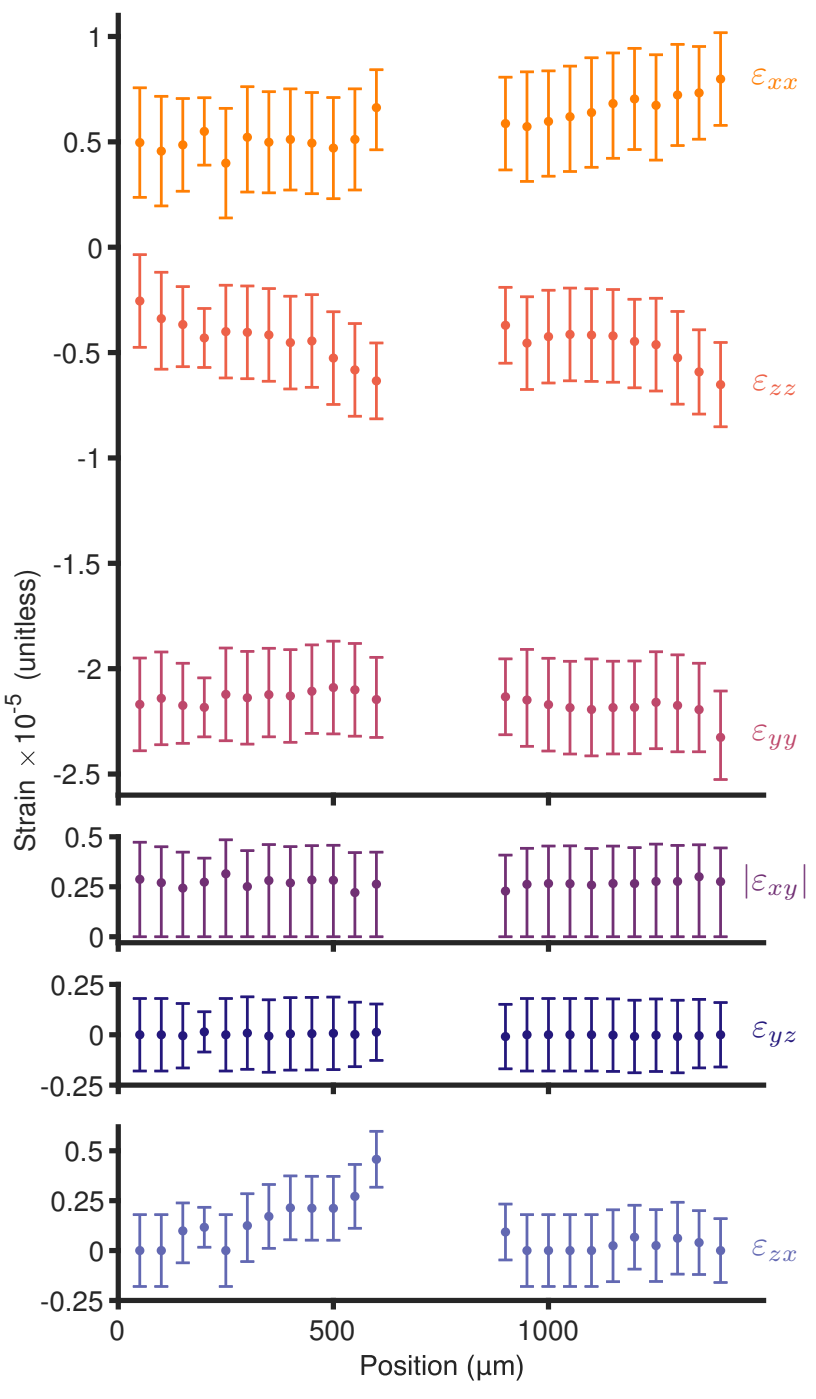

FIG. 4. Calculated strain tensor indices across the points shown in Fig. 3 The first plot shows the normal strain tensor indices, $\varepsilon_{x x}$, $\varepsilon_{y y}$, and $\varepsilon_{z z}$. The following three plots show the sheer strain tensor indices, $\varepsilon_{x y}$ (for which positive values are plotted due to a sign ambiguity), $\varepsilon_{y z}$ and $\varepsilon_{z x} .90 \%$ confidence intervals are shown.

an ensemble of $\mathrm{SiV}^{-}$centers, as mentioned in Section IIA This means we measure the strain tensor averaged over the laser spot, rather than at specific centers. Note that this variation in macroscopic strain is also different from the microscopic strain fluctuations proposed in Ref. 28, as the latter concerns individual $\mathrm{SiV}^{-}$centers in highly strained environments.

The source of this strain is likely due to the implantation and annealing processes. As mentioned in Section IIB, our sample has a rather high number density of implanted silicon of $7.5 \times 10^{18} \mathrm{~cm}^{-3}$. This density corresponds to about one silicon atom for every $2.3 \times 10^{4}$ carbon atoms. If we assume, as an approximation, implanting the silicon does not increase the size of the sample, but merely pushes the atoms uniformly closer together, then we can estimate the normal strain as $-1.4 \times 10^{-5}$. While this simple estimation does not take into account many complexities of the system, it does agree with our experimental values to within an order of magnitude.

\section{CONCLUSION}

By analyzing both MDCS and TPC measurements of $\mathrm{SiV}^{-}$ centers, we were able to measure the full strain tensor of our sample. We do measure a nonzero strain, which varies across the sample. This strain is most likely due to the large amount of implanted silicon in our sample. In future work, we hope to take measurements on a variable density sample, to further measure the relationship between implantation density and strain. This work may also be useful in using $\mathrm{SiV}^{-}$centers in diamond as a high pressure strain gauge, potentially in a diamond anvil cell.

\section{ACKNOWLEDGEMENTS}

T. S. acknowledges support from the Federal Ministry of Education and Research of Germany (BMBF, project DiNOQuant, 13N14921). Ion implantation work to generate the $\mathrm{SiV}^{-}$centers was performed, in part, at the Center for Integrated Nanotechnologies, an Office of Science User Facility operated for the U.S. Department of Energy (DOE) Office of Science. Sandia National Laboratories is a multi-mission laboratory managed and operated by National Technology and Engineering Solutions of Sandia, LLC., a wholly owned subsidiary of Honeywell International, Inc., for the DOE's National Nuclear Security Administration under contract DENA-0003525. This paper describes objective technical results and analysis. Any subjective views or opinions that might be expressed in the paper do not necessarily represent the views of the DOE or the United States Government.

${ }^{1}$ J. M. Taylor, P. Cappellaro, L. Childress, L. Jiang, D. Budker, P. R. Hemmer, A. Yacoby, R. Walsworth, and M. D. Lukin, "High-sensitivity diamond magnetometer with nanoscale resolution," Nat. Phys. 4, 810-816 (2008) arXiv:0805.1367

${ }^{2}$ K. Jensen, N. Leefer, A. Jarmola, Y. Dumeige, V. M. Acosta, P. Kehayias, B. Patton, and D. Budker, "Cavity-enhanced room-temperature magnetometry using absorption by nitrogen-vacancy centers in diamond," Phys. Rev. Lett. 112, 160802 (2014)

${ }^{3}$ T. Wolf, P. Neumann, K. Nakamura, H. Sumiya, T. Ohshima, J. Isoya, and J. Wrachtrup, "Subpicotesla diamond magnetometry," Phys. Rev. X 5, 041001 (2015)

${ }^{4}$ M. J. H. Ku, T. X. Zhou, Q. Li, Y. J. Shin, J. K. Shi, C. Burch, L. E. Anderson, A. T. Pierce, Y. Xie, A. Hamo, U. Vool, H. Zhang, F. Casola, T. Taniguchi, K. Watanabe, M. M. Fogler, P. Kim, A. Yacoby, and R. L. Walsworth, "Imaging viscous flow of the dirac fluid in graphene," Nature 583, 537-541 (2020)

${ }^{5}$ V. Ivády, J. Davidsson, N. T. Son, T. Ohshima, I. A. Abrikosov, and A. Gali, "Identification of si-vacancy related room-temperature qubits in $4 h$ silicon carbide," Phys. Rev. B 96, 161114 (2017)

${ }^{6}$ C. Kurtsiefer, S. Mayer, P. Zarda, and H. Weinfurter, "Stable solid-state source of single photons,"'Phys. Rev. Lett. 85, 290-293 (2000)

${ }^{7}$ A. Beveratos, R. Brouri, T. Gacoin, J.-P. Poizat, and P. Grangier, "Nonclassical radiation from diamond nanocrystals," Phys. Rev. A 64, 061802 (2001) 
${ }^{8}$ L. J. Rogers, K. D. Jahnke, M. W. Doherty, A. Dietrich, L. P. McGuinness, C. Müller, T. Teraji, H. Sumiya, J. Isoya, N. B. Manson, and F. Jelezko, "Electronic structure of the negatively charged silicon-vacancy center in diamond," Phys. Rev. B 89, 235101 (2014)

${ }^{9}$ A. Sipahigil, K. D. Jahnke, L. J. Rogers, T. Teraji, J. Isoya, A. S. Zibrov, F. Jelezko, and M. D. Lukin, "Indistinguishable photons from separated silicon-vacancy centers in diamond," Phys. Rev. Lett. 113, 113602 (2014)

${ }^{10}$ R. Budnik, O. Cheshnovsky, O. Slone, and T. Volansky, "Direct detection of light dark matter and solar neutrinos via color center production in crystals," Phys. Lett. B 782, 242-250 (2018)

${ }^{11}$ S. Rajendran, N. Zobrist, A. O. Sushkov, R. Walsworth, and M. Lukin, "A method for directional detection of dark matter using spectroscopy of crystal defects," Phys. Rev. D 96, 035009 (2017)

${ }^{12}$ P. Udvarhelyi, R. Nagy, F. Kaiser, S.-Y. Lee, J. Wrachtrup, and A. Gali, "Spectrally stable defect qubits with no inversion symmetry for robust spinto-photon interface,"' Phys. Rev. Applied 11, 044022 (2019)

${ }^{13}$ A. A. Gorokhovsky, A. V. Turukhin, R. R. Alfano, and W. Phillips, "Photoluminescence vibrational structure of si center in chemicalvapor deposited diamond," Appl. Phys. Lett 66, 43-45 (1995) https://doi.org/10.1063/1.114176

${ }^{14}$ E. Neu, D. Steinmetz, J. Riedrich-Möller, S. Gsell, M. Fischer, M. Schreck, and C. Becher, "Single photon emission from silicon-vacancy colour centres in chemical vapour deposition nano-diamonds on iridium," New J. Phys. 13, 025012 (2011) arXiv: 1008.4736

${ }^{15}$ B. C. Rose, D. Huang, Z.-H. Zhang, P. Stevenson, A. M. Tyryshkin, S. Sangtawesin, S. Srinivasan, L. Loudin, M. L. Markham, A. M. Edmonds, D. J. Twitchen, S. A. Lyon, and N. P. de Leon, "Observation of an environmentally insensitive solid-state spin defect in diamond," Science 361, 60-63 (2018) https://science.sciencemag.org/content/361/6397/60.full.pdf

${ }^{10}$ K. D. Jahnke, A. Sipahigil, J. M. Binder, M. W. Doherty, M. Metsch, L. J. Rogers, N. B. Manson, M. D. Lukin, and F. Jelezko, "Electron-phonon processes of the silicon-vacancy centre in diamond," New J. Phys. 17, 043011 (2015) arXiv:1411.2871

${ }^{1 /}$ J. N. Becker, B. Pingault, D. Groß, M. Gündoğan, N. Kukharchyk, M. Markham, A. Edmonds, M. Atatüre, P. Bushev, and C. Becher, "Alloptical control of the silicon-vacancy spin in diamond at millikelvin temperatures," Phys. Rev. Lett. 120, 053603 (2018)
${ }^{18}$ J. Cai, F. Jelezko, and M. B. Plenio, "Hybrid sensors based on colour centres in diamond and piezoactive layers," Nat. Commun. 5, 4065 (2014)

${ }^{19}$ M. E. Trusheim and D. Englund, "Wide-field strain imaging with preferentially aligned nitrogen-vacancy centers in polycrystalline diamond," New J. Phys. 18, 123023 (2016)

${ }^{20}$ S. Meesala, Y.-I. Sohn, B. Pingault, L. Shao, H. A. Atikian, J. Holzgrafe, M. Gündoğan, C. Stavrakas, A. Sipahigil, C. Chia, R. Evans, M. J. Burek, M. Zhang, L. Wu, J. L. Pacheco, J. Abraham, E. Bielejec, M. D. Lukin, M. Atatüre, and M. Lončar, "Strain engineering of the silicon-vacancy center in diamond," Phys. Rev. B 97, 205444 (2018)

${ }^{21}$ A. Jayaraman, "Diamond anvil cell and high-pressure physical investigations," Rev. Mod. Phys. 55, 65-108 (1983)

${ }^{22} \mathrm{~S}$. T. Cundiff and S. Mukamel, "Optical multidimensional coherent spectroscopy," Phys. Today 66, 44 (2013)

${ }^{23}$ C. L. Smallwood and S. T. Cundiff, "Coherent spectroscopy: Multidimensional coherent spectroscopy of semiconductors," Laser Photonics Rev. 12, 1870052 (2018) https://onlinelibrary.wiley.com/doi/pdf/10.1002/lpor.201870052

${ }^{24}$ P. F. Tekavec, G. A. Lott, and A. H. Marcus, "Fluorescence-detected two-dimensional electronic coherence spectroscopy by acoustooptic phase modulation," J. Chem. Phys. 127, 214307 (2007) https://doi.org/10.1063/1.2800560

${ }^{25}$ G. Nardin, T. M. Autry, K. L. Silverman, and S. T. Cundiff, "Multidimensional coherent photocurrent spectroscopy of a semiconductor nanostructure," Opt. Express 21, 28617-28627 (2013)

${ }^{26} \mathrm{~K}$. Momma and F. Izumi, "VESTA3 for three-dimensional visualization of crystal, volumetric and morphology data," J. Appl. Crystallogr. 44, 1272$1276(2011)$

${ }^{27}$ C. D. Clark, H. Kanda, I. Kiflawi, and G. Sittas, "Silicon defects in diamond," Phys. Rev. B 51, 16681-16688 (1995)

${ }^{28}$ C. L. Smallwood, R. Ulbricht, M. W. Day, T. Schröder, K. M. Bates, T. M. Autry, G. Diederich, E. Bielejec, M. E. Siemens, and S. T. Cundiff, "Hidden silicon-vacancy centers in diamond," (2021), arXiv:2006.02323 [quant$\mathrm{ph}]$

${ }^{29}$ M. W. Day, K. M. Bates, C. L. Smallwood, , R. C. Owen, R. Ulbricht, T. Schröder, E. Bielejec, and S. T. Cundiff, "Quantum coherent interactions between silicon-vacancy centers in diamond," (2021), in progress. 